\title{
Testing of laser components subjected to exposure in space
}

\author{
Narasimha S. Prasad* \\ NASA Langley Research Center, 5 N. Dryden St., MS 468, Hampton VA, 23681
}

\begin{abstract}
Materials International Space Station Experiment (MISSE) missions provide an opportunity for developing space qualifiable materials by studying the response of novel materials when subjected to the synergistic effects of the harsh space environment. MISSE 6 was transported to the international Space Station (ISS) via STS 123 on March 11. 2008. The astronauts successfully attached the passive experiment containers (PEC) to external handrails of the international space station (ISS) and opened up for long term exposure. After more than a year of exposure attached to the station's exterior, the PEC with several hundred material samples returned to the earth with the STS-128 space shuttle crew that was launched on shuttle Discovery from the Kennedy Space Center, Fla., on Aug. 28. Meanwhile, MISSE 7 launch is scheduled to be launched on STS 129 mission. MISSE-7 was launched on Space Shuttle mission STS-129 on Atlantis was launched on November 16, 2009. This paper will briefly review recent efforts on MISSE 6 and MISSE 7 missions at NASA Langley Research Center (LaRC).
\end{abstract}

Keywords: MISSE 6, MISSE 7, Space Qualification, Laser components, International Space Station, STS-123, STS 129

\section{INTRODUCTION}

The goal of MISSE program is to evaluate the performance, stability, and long-term survivability of materials and components planned for use by NASA, Department of Defense (DOD), other federal agencies and private entities on future Low Earth Orbit (LEO), synchronous orbit, and interplanetary space missions. The study of combined effects of radiation, temperature cycling, ultraviolet (UV) light and atomic oxygen in space environment due to long term exposures will help in developing space qualifiable elements for future space missions. In-situ space testing is critical since it is difficult and not economical to conduct these studies inside simulated terrestrial facilities. The development of new generations of materials will allow our nation to continue maintain technological superiority related to space endeavors.

NASA's Langley Research Center (LaRC), Hampton, Virginia has managed six MISSE projects. Other NASA Centers participating in this project include Glenn Research Center, Cleveland; Goddard Space Flight Center, Greenbelt, Maryland, Johnson Space Center, Houston, Texas, the Jet Propulsion Laboratory, Pasadena, California, and Marshall Space Flight Center, Huntsville, Alabama. Several DOD and industry partners including the Boeing Company have been involved with this effort. MISSE program is a direct successor of the Mir Environmental Effects Payloads (MEEP) that were attached for over a year to the Mir Docking Module of the space station Mir between shuttle flights STS-76 and STS- $86^{1}$.

\section{AN OVERVIEW OF THE MISSE PROGRAM}

MISSE is a series of experiments and so far, six MISSE missions have been successfully completed. More than 1500 samples have been tested on the MISSE project. Samples include chemicals, sensor devices, opto-mechanical elements, polymers, coatings, and biological materials and species, composites. The MISSE project has also provided educational opportunities for students. MISSEs 3 and 4 have transported approximately 8 million basil seeds for science experiments of children to stimulate interest in space science.

A suitcase shaped rugged flight-worthy box known as Passive Experiment Containers (PECs) is used to transport the selected materials to and from the ISS. PECs were originally developed and used by NASA's LaRC, Hampton, Virginia, for ISS Phase I Risk Mitigation Program experiments conducted on the Russian Mir space station. Specific steps required for transporting materials include specimen preparation, fixing specimens inside a holder, integration of specimen holder on a tray, integration of trays in a PEC, integration of PEC inside a carrier for launch survivability tests

\footnotetext{
*narasimha.s.prasad@nasa.gov; Phone 757-864-9403; Fax 757-864-8828.
} 
and ease of transport. During space walk, also known as extra-vehicular activity (EVA) (i.e., when an astronaut works outside of a spacecraft), the PECs are attached to the handrails or at a specific location that is exposed to space environment. After exposure in space, MISSE PEC is retrieved in the same manner as it was deployed. MISSE PECs have active and passive detectors to give a time-history reading, or a reading of what happened to the materials at certain points in time. The passive detectors report a cumulative measure of the following environments that the test specimens are exposed to namely UV exposure, atomic oxygen exposure, molecular contamination, tray temperature and man-made debris. Back on the ground, tests will be conducted to determine the effects of its exposure for several months in space. The material samples are tested in laboratory conditions to see if they still possess their unique properties needed to complete space missions. The details of MISSE 1 to MISSE 6 missions can be found at NASA's MISSE webpage ${ }^{2}$. In this paper, the status of MISSE 6 mission followed by optical components packaged by NASA LaRC for MISSE 7 mission are discussed.

\section{SELECTED ITEMS FOR THE MISSE 6 MISSION}

Among several specimens from NASA Langley Research Center, optical and laser related components including waveguide laser components, ceramic yttria and $\mathrm{PbTe}$ based thermoelectric material were included on a sub-assembly. MISSE 6 package had two sections namely MISSE 6A and MISSE 6B. The specimen holder from LaRC was located on MISSE 6B PEC oriented for UV exposure.

\subsection{Optical elements on a specimen holder}

Figure 1 shows the optical items mounted on a specimen holder. The laser diode, diode bar, ceramic yttria ${ }^{3}$ and Bismuth doped PbTe are visible. Other elements are below a secondary plate and are hidden from the direct view. The unique, patented waveguide gain media ${ }^{4}$ offers additional capabilities for space applications, such as amplifiers and pump sources for nonlinear optical devices. Figure 2 illustrates fully integrated PEC with specimen trays mounted on a carrier and located on a pre-launch test platform.
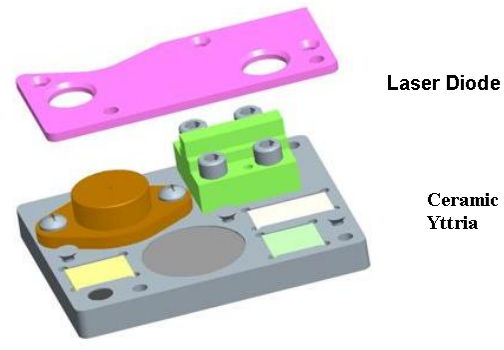

Fig. 2. The PEC with carrier on a test platform.

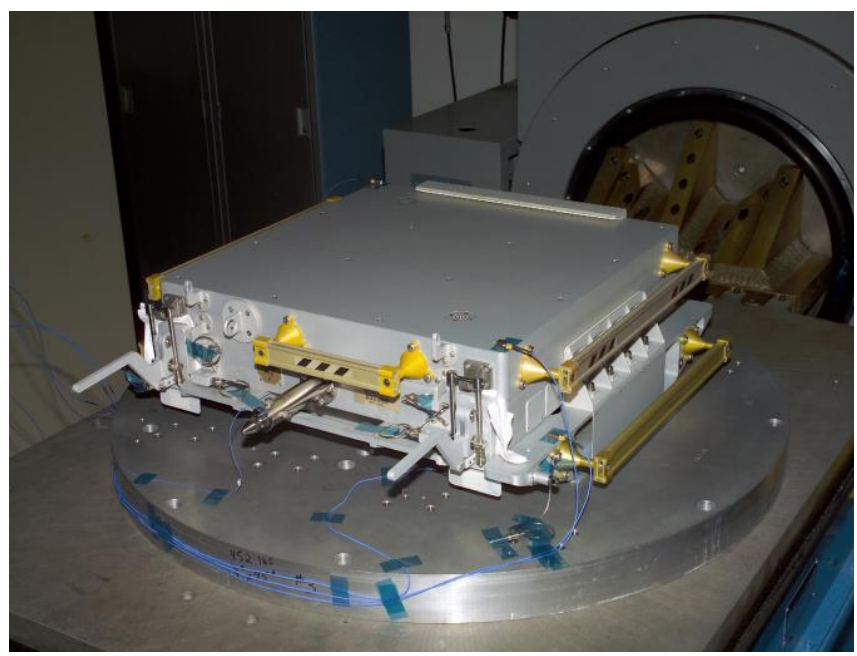




\section{DEPLOYMENT AND RETRIEVAL OF MISSE 6 PEC}

The MISSE 6 PEC was transported on the STS-123 Mission to ISS on March 11, 20085,. Following five spacewalks, astronauts installed the MISSE 6 on the outside of the European Agency's Columbus module. Figure 3 shows the MISSE 6 PEC attached to the Columbus module on the ISS. The MISSE-6A and 6B investigators are studying more than 400 new materials. After more than a year of exposure attached to the station's exterior, MISSE 6 PEC with all materials returned to the earth with the STS-128 space shuttle crew that was launched on shuttle Discovery from the Kennedy Space Center, Fla., on Aug. 28. During a spacewalk on Sept. 1, the STS-128 astronauts Danny Olivas and Nicole Stott removed MISSE-6A and 6B from outside the Columbus Laboratory ${ }^{7}$. On October 20, 2009, the retrieved PEC was opened at NASA LaRC as illustrated in Figure 4. All specimens were removed from the PEC tray and distributed to respective investigators for post-characterization. In Figure 5, the retrieved specimen holder with optical components from NASA LaRC is shown. The visual inspection of specimens did not show any signs of external damage. The specimens are now undergoing tests.
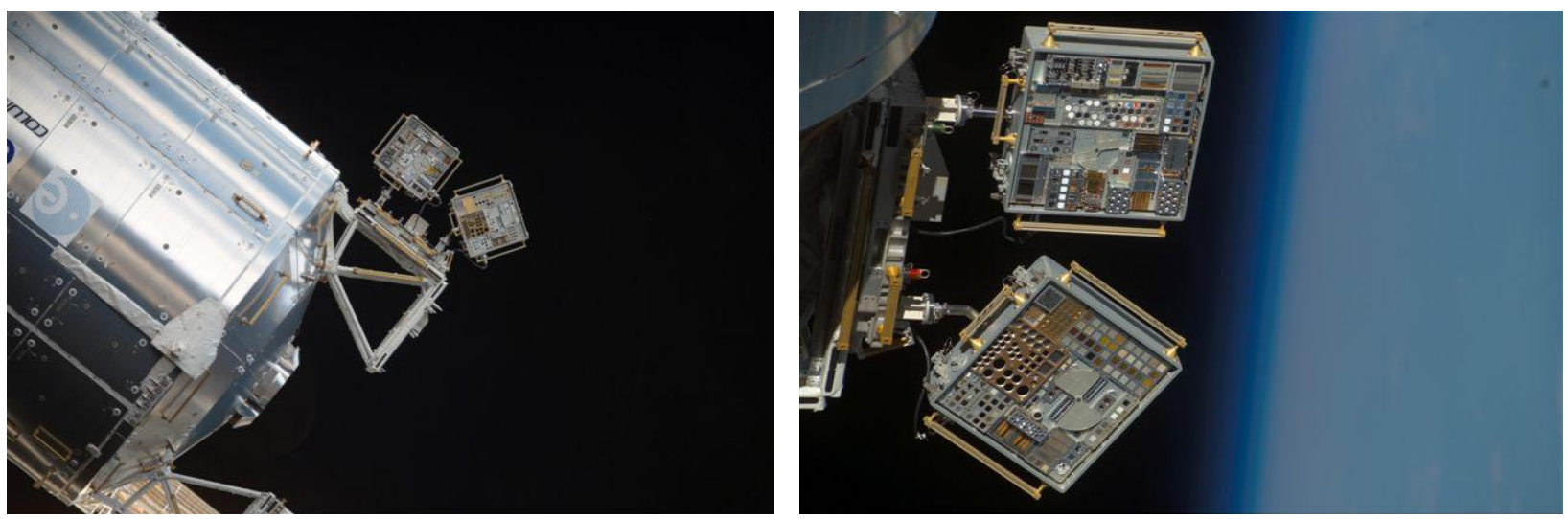

Fig. 3. (Left) The PEC attached to the Columbus module on ISS. (Right) A close-up view of MISSE PEC in space.
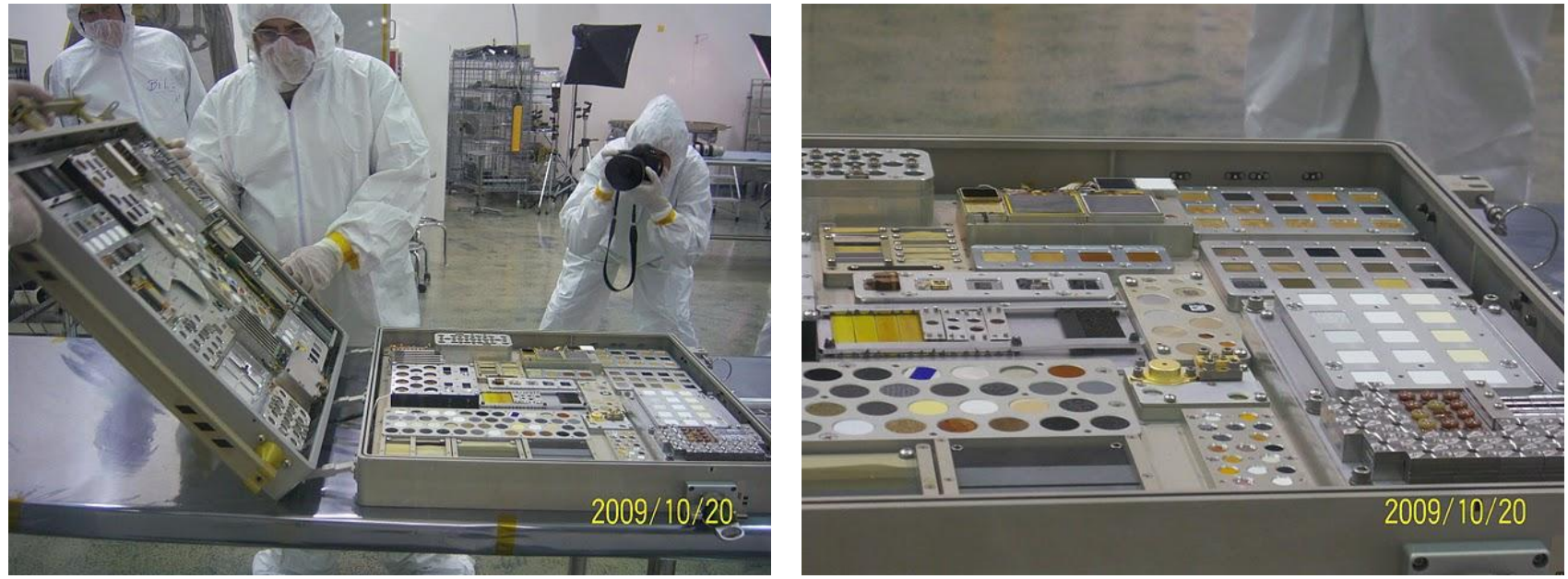

Figure 4. Unpacking of retrieved MISSE 6 PEC at NASA LaRC 

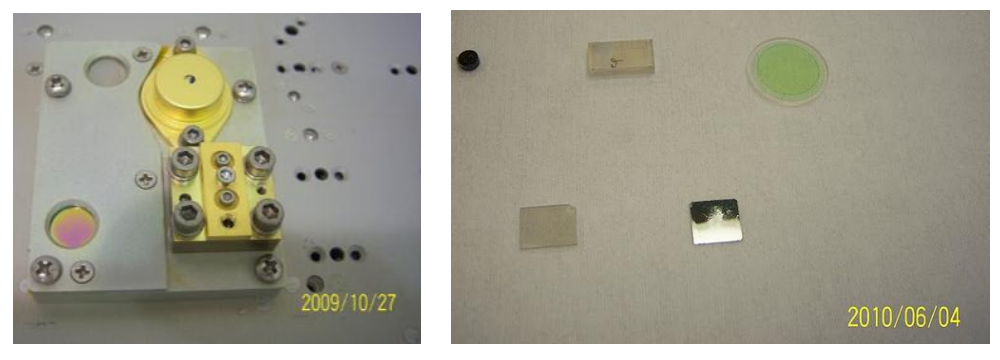

Figure 5. (Left) Retrieved specimen holder and (Right) optical specimens

\section{THE MISSE 7 MISSION- A BRIEF OVERVIEW}

The MISSE 7 mission is being managed by Glenn Research Center with Naval Research Laboratory (NRL), Boeing and NASA LaRC playing significant roles. Boeing was selected to be the platform integrator. The MISSE 7 efforts were accelerated in 2008. Items from various research groups were sent to Naval Research Laboratory (NRL), Washington, DC for platform integration and testing. The MISSE 7 PEC is a suite of experiments that include over 700 new and affordable materials for potential space applications. In the case of MISSE 7 package, there were two sections, 7A and 7B. NASA LaRC provided PECs and a flight worthy package with laser and lidar components along with other logistics support

MISSE 7 was launched on Space Shuttle mission STS-129 ${ }^{8,9}$. MISSE 7A and 7B trays were attached outside the ISS on the EXPRESS Logistics Carrier 2 (ECL2) on the S3 truss, and then opened and exposed to space during a spacewalk on November, 23, 2009. PEC 7A's orientation is zenith/nadir (space facing/Earth facing) while PEC 7B faces ram/wake (forward/backward) relative to the ISS orbit. Power and data is being provided by the ISS. MISSE 7 will use the ISS communication system uplink/downlink capabilities to receive commands to downlink data. No crew interaction is required for data transfer operations. MISSE 7 is planned to be retrieved on STS 134 in late 2010 or early 2011.

NASA LaRC has sent a package with several lidar transceiver components for operation in near-IR spectral band as shown in Figure $6^{10}$. These components include 1.5 micron fiber laser, high power 808 nm laser diode bar, fiber coupled

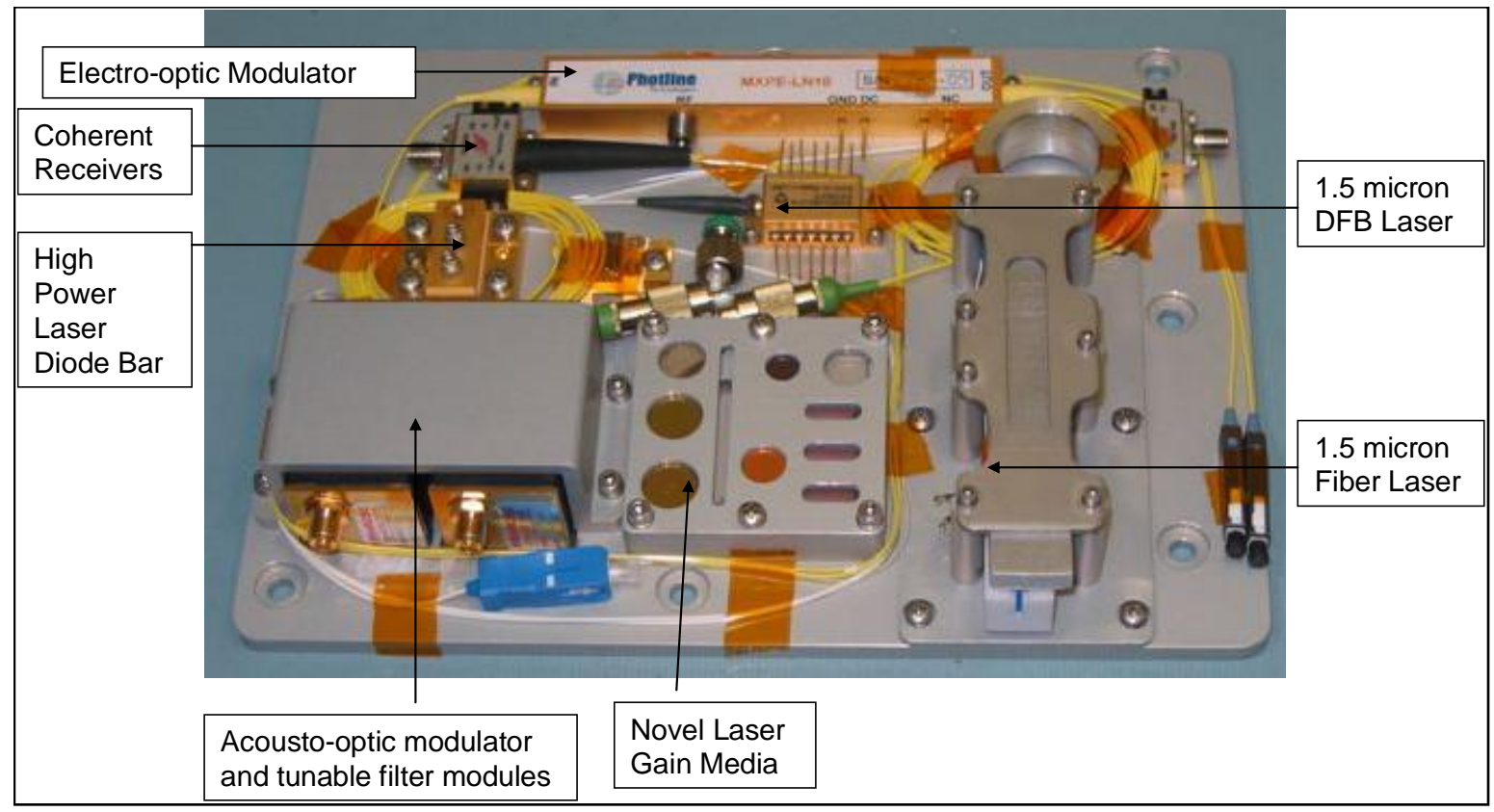

Figure 6. Lidar transceiver components assembled on a plate. 


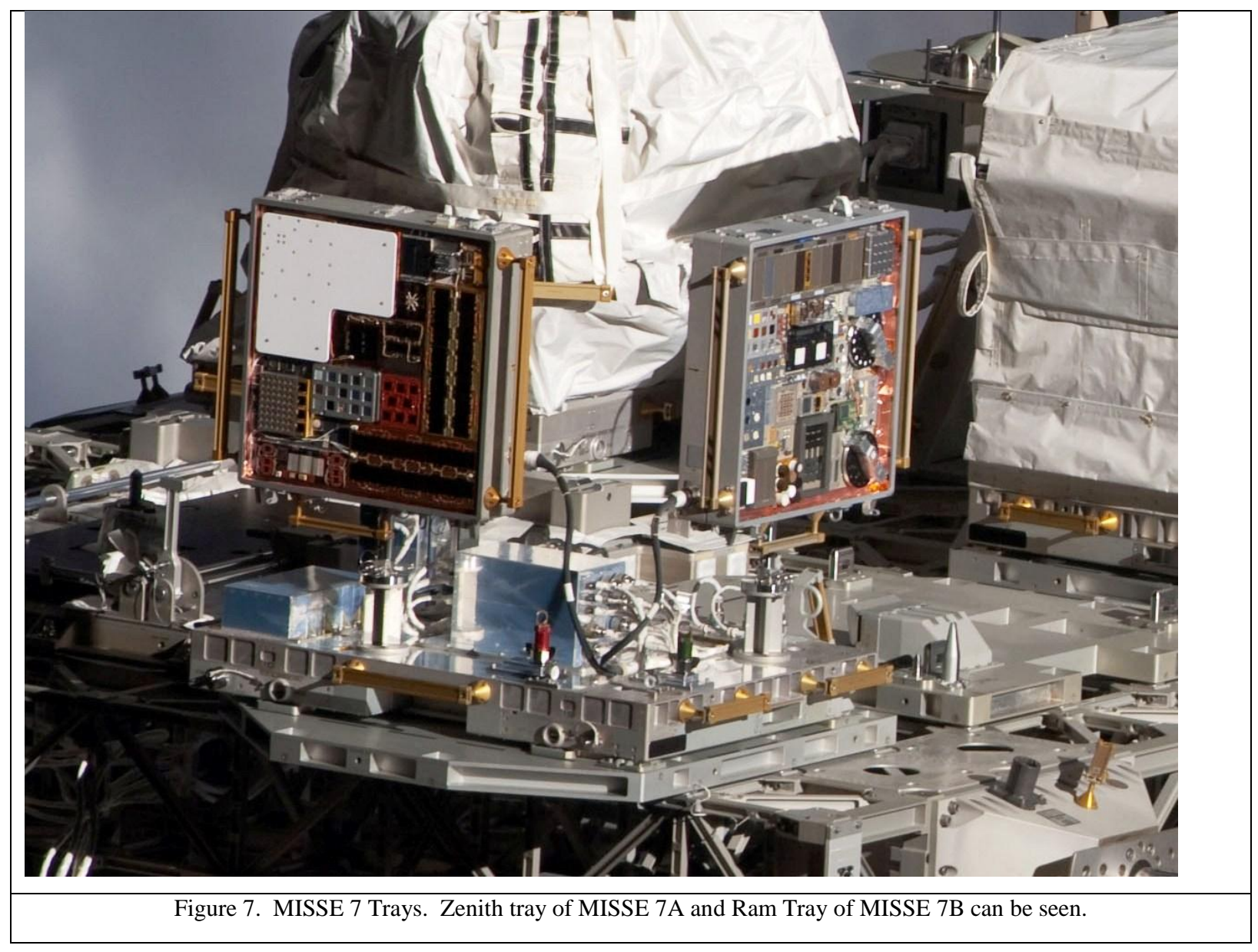

distributed feedback (DFB) laser, tellurium dioxide $\left(\mathrm{TeO}_{2}\right)$ based acousto-optic modulator and tunable filter, coherent receivers, and several types of acousto-optic and laser gain media including mercurous chloride, mercurous bromide, gallium phosphide, chromium doped zinc selenide (Cr:ZnSe), erbium doped ytterbium aluminum garnet (Er:YAG) and erbium doped lutetium aluminum garnet (Er:LuAG) crystals. Figure 6 shows the laboratory tested components packed on a flight qualified platform. This package will be mounted on MISSE 7 PEC facing wake side investigations primarily for UV and radiation exposure effects. Figure 7 shows platform integration and testing being done at NRL ${ }^{7}$.

\section{SUMMARY AND CONCLUSIONS}

The objective of the MISSE program is to study the performance, stability, and long-term survivability of novel materials when subjected to the synergistic effects of the harsh space environment. So far, six MISSE missions have been completed. The MISSE 6 PEC was transported on March 11, 2008 on STS-123 mission and was retrieved by STS128 mission. The MISSE 6 PEC returned to the Earth on August 28, 2009. The MISSE 7 PEC was transported in November 2009 on STS-129 mission and will return to the earth in late 2010 or early 2011 on STS 134 mission. Several optical specimens including waveguide laser components, laser diodes, thermoelectric power generator materials and ceramic yttria specimens were returned to Earth after predominantly UV exposure for more than a year on the ISS. Several specimens and devices for use in Laser and Lidar systems were selected from NASA LaRC for MISSE 7 mission. Upon retrieval, these specimens will undergo similar tests to look into any possible deviations from preexposure characteristics. 


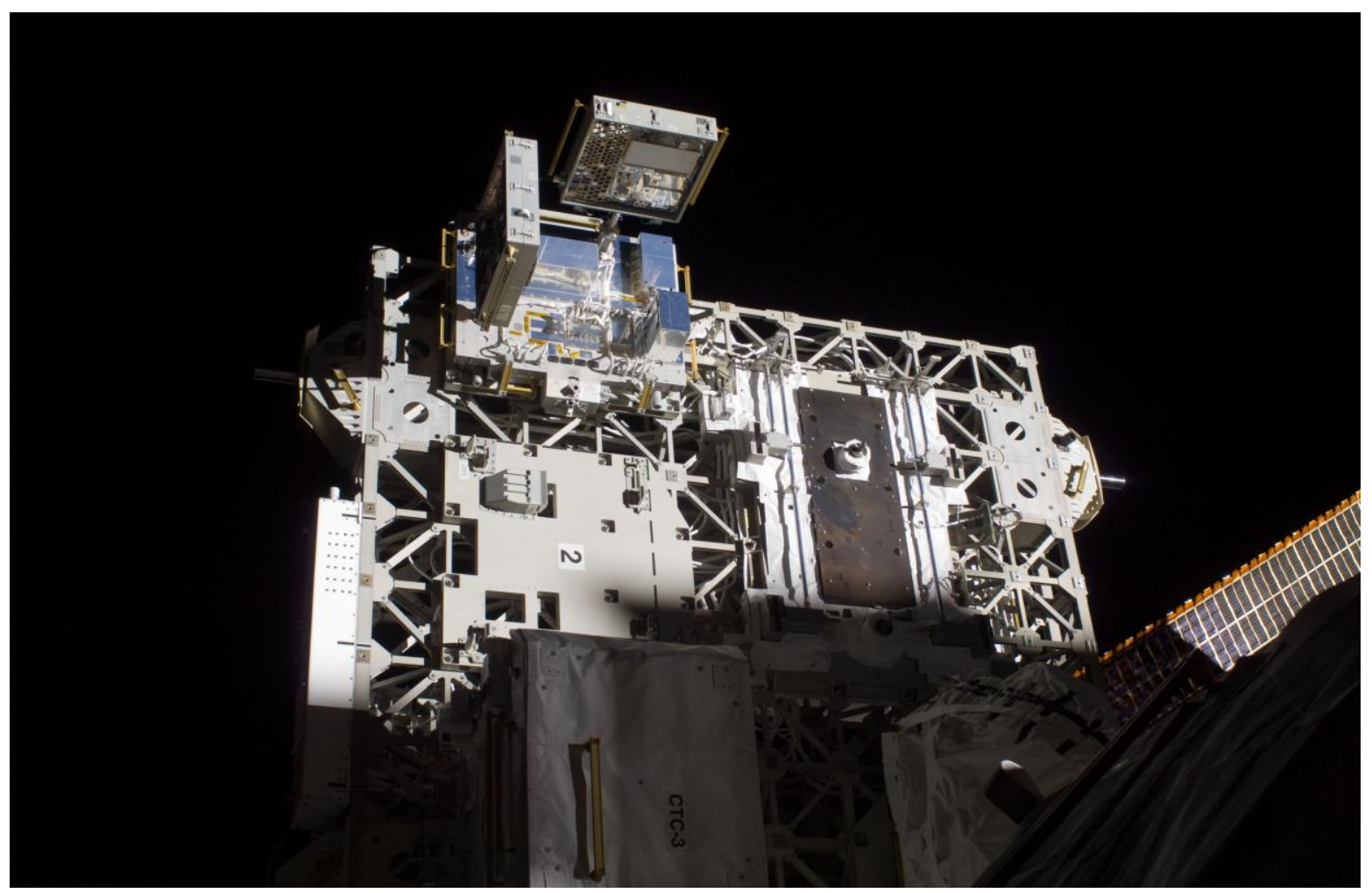

Figure 7. The orientation of MISSE 7 PEC trays on ISS in orbit.

\section{ACKNOWLEDGEMENTS}

The author acknowledges William H. Kinard for providing guidance and support in the initial stages of MISSE 7 mission development. The author is grateful to William C. Edwards for arranging funds for this effort. Finally, the author thanks Karen Gibson, Terry Clark and John Chapman for mechanical engineering and flight package quality control efforts.

\section{REFERENCES}

[1] William H. Kinard, "MIR Environmental Effects Payload (MEEP) Archive System," NASA, Langley Research Center, Hampton, Virginia. (http://setas-www.larc.nasa.gov/meep/meep.html and http://www.nasa.gov/centers/langley/news/factsheets/misse_2005.html)

[2] The NASA MISSE website: http://misseone.larc.nasa.gov/

[3] Prasad, N.S.; Edwards, W.C.; Trivedi, S.B.; Kutcher, S.W.; Chen-Chia Wang; Joo-Soo Kim; Hommerich, U.; Shukla, V.; Sadangi, R.; Kear, B.H., "Recent Progress in the Development of Neodymium-Doped Ceramic Yttria," Selected Topics in IEEE Journal of Quantum Electronics, Vol. 13, Issue 3, 831 - 837(2007).

[4] US Patent Number 6,894,828, "Power scalable waveguide amplifier and laser device"(2005).

[5] STS-123 MCC Status Report \#15 (http://www.nasa.gov/mission_pages/shuttle/shuttlemissions/sts123/news/STS123-15.html).

[6] STS-123 MCC Status Report \#25 (http://www.nasa.gov/mission_pages/shuttle/shuttlemissions/sts123/news/STS123-25.html).

[7] http://www.nasa.gov/mission_pages/station/science/misse2009.html

[8] http://www.nasa.gov/mission_pages/station/science/misse.html

[9] http://www.nasa.gov/mission_pages/station/science/experiments/MISSE-7.html

[10] Narasimha S. Prasad, "Testing of NASA LaRC materials under MISSE 6 and MISSE 7 missions," Proc. SPIE vol. 7614, 2009. 